\title{
PARTIAL ACTIONS OF INVERSE AND WEAKLY LEFT $E$-AMPLE SEMIGROUPS
}

\author{
VICTORIA GOULD and CHRISTOPHER HOLLINGS ${ }^{\bowtie}$
}

(Received 22 August 2007; accepted 11 December 2007)

\author{
Communicated by M. G. Jackson
}

\begin{abstract}
We introduce partial actions of weakly left E-ample semigroups, thus extending both the notion of partial actions of inverse semigroups and that of partial actions of monoids. Weakly left $E$-ample semigroups arise very naturally as subsemigroups of partial transformation semigroups which are closed under the unary operation $\alpha \mapsto \alpha^{+}$, where $\alpha^{+}$is the identity map on the domain of $\alpha$. We investigate the construction of 'actions' from such partial actions, making a connection with the FA-morphisms of Gomes. We observe that if the methods introduced in the monoid case by Megrelishvili and Schröder, and by the second author, are to be extended appropriately to the case of weakly left $E$-ample semigroups, then we must construct not global actions, but so-called incomplete actions. In particular, we show that a partial action of a weakly left $E$-ample semigroup is the restriction of an incomplete action. We specialize our approach to obtain corresponding results for inverse semigroups.
\end{abstract}

2000 Mathematics subject classification: primary 20M30; secondary 20M18.

Keywords and phrases: augmented action, expansion, incomplete action, inverse semigroup, partial action, premorphism, weakly left $E$-ample semigroup.

\section{Introduction}

Partial monoid actions were introduced by Megrelishvili and Schröder in [22] as a generalization of the partial group actions of Kellendonk and Lawson [17]. The authors of [22] considered the partial actions of monoids first on sets and then on topological and metric spaces. In each instance, they demonstrated that such partial actions can always be globalized, that is, given the partial action of a monoid $M$ on a set/space $X$, we can always construct a larger set/space $Y$ upon which $M$ acts globally. Consequently, monoid partial actions are precisely restrictions (in an appropriate sense) of global actions, a fact which indicates both their importance and the naturalness of the concept.

The study of the partial actions of monoids was taken up in [13], in which it was shown that the partial monoid actions of Megrelishvili and Schröder (termed strong

(C) 2009 Australian Mathematical Society 1446-7887/2009 \$16.00 
partial actions) are equivalent to a class of mappings called strong premorphisms, a notion adapted from the work of Kellendonk and Lawson [17] in the group case. This equivalence is analogous to that between morphisms and global actions. In terms of strong premorphisms, Megrelishvili and Schröder's result on globalization can be phrased thus: given a strong premorphism $\theta: M \rightarrow \mathcal{P} \mathcal{T}_{X}$ (where $\mathcal{P} \mathcal{T}_{X}$ is the partial transformation monoid on the set $X$ ), we can always find a larger set $Y \supseteq X$ and a monoid morphism $\varphi: M \rightarrow \mathcal{T}_{Y}$ such that $s \theta=\iota(s \varphi) \iota^{-1}$, for each $s \in M$, where $\iota: X \rightarrow Y$ is inclusion. Conversely, given a monoid morphism $\varphi$ : $M \rightarrow \mathcal{T}_{Y}$, we can 'restrict' $\varphi$ to any subset $X$ of $Y$ to obtain a strong premorphism $\theta: M \rightarrow \mathcal{P} \mathcal{T}_{X}$.

In the group case, Kellendonk and Lawson [17], and independently Exel [4], present a second method for constructing an action from a partial action: the expansion method, in which the set being acted upon is fixed, whilst the group is modified by taking an expansion, in the sense of Birget and Rhodes [3]. This method has subsequently been generalized to the monoid case in [13]. In both [17] and [13], the expansion of choice was the Szendrei expansion of [7, 30].

The partial transformation monoid $\mathcal{P} \mathcal{T}_{X}$ is a weakly left E-ample semigroup, where the ' $E$ ' refers to a particular semilattice (in this case, the semilattice of partial identities on $X$; for details, see Section 2 ). Indeed, any weakly left $E$-ample semigroup $S$ is a $(2,1)$-subalgebra of $\mathcal{P} \mathcal{T}_{S}$, where the unary operation is ${ }^{+}: \alpha \mapsto I_{\operatorname{dom} \alpha}$ [12]. In the case where $E=E(S)$ we say that $S$ is weakly left ample. Weakly left $E$-ample semigroups appear numerous times in the literature, under a variety of names. We believe the first instance to be as reducts of the function systems of Schweizer and Sklar, which were developed through a series of papers [26-29] in the 1960s. This class of semigroups was touched upon again in [24], but the first person to study weakly left $E$-ample semigroups proper was Trokhimenko in [31]; in particular, he was the first to prove that they may be represented as systems of partial mappings (see our Theorem 2.2). Weakly left $E$-ample semigroups appeared also as the type SL2 $\gamma$ semigroups of Batbedat [1,2] in the early 1980s. More recently, they have arisen in the work of Jackson and Stokes [15] in the guise of (left) twisted C-semigroups and in that of Manes [20] as guarded semigroups, motivated by consideration of closure operators and categories, respectively. Our terminology 'weakly left $E$-ample' was first used in [8], arrived at from the starting point of the left ample semigroups of Fountain [5, 6] via the route of replacing considerations of the relation $\mathcal{R}^{*}$ on a semigroup $S$ by those of $\widetilde{\mathcal{R}}$ (hence the 'weakly') and by making reference to a specific set of idempotents $E$ (which may not be the whole of $E(S)$ ).

Inverse semigroups are clearly weakly left ample, as are left ample semigroups. Any monoid is weakly left $\{1\}$-ample; the study of strong partial monoid actions thus far has therefore been concerned with strong unitary premorphisms from weakly left $\{1\}$-ample semigroups to arbitrary weakly left $E$-ample monoids. The aim of this paper is to develop the theory for strong premorphisms between arbitrary weakly left $E$ ample semigroups $S$ and $T$ (the specialization to monoids being no longer significant), and thus to consider the strong partial actions of $S$. 
Note that the term 'partial action' has two slightly different uses in the literature; compare, for example, the definition of 'partial action' in [23] with that in [13]. The sense in which we will use the term derives ultimately from [17] via [22] and [13]. Loosely speaking, our partial actions are 'partial' in two senses: first, they are not defined everywhere; and second, they are equivalent to premorphisms, rather than morphisms. We will also make use of the other notion of 'partial action' but, in the interests of clarity, we will call such an action an incomplete action.

Recall that the globalization of the partial action of a monoid $M$ on a set $X$ calls for the construction of a monoid morphism $\varphi: M \rightarrow \mathcal{T}_{Y}$. In order to extend this construction to arbitrary weakly left $E$-ample semigroups, the condition that $1 \varphi=1$ is naturally replaced by $a^{+} \varphi=(a \varphi)^{+}$; this introduces extra complications which require new techniques. As in the inverse case, if this construction is to be achieved, then we must allow $\varphi$ to have codomain $\mathcal{P} \mathcal{T}_{Y}$, rather than $\mathcal{T}_{Y}$. Such a $(2,1)$-morphism is not equivalent to a global action, but to an incomplete action. In the case of the partial action of a weakly left $E$-ample semigroup $S$, we modify Megrelishvili and Schröder's notion of 'globalization' so that we construct a (2,1)-morphism $\varphi: S \rightarrow \mathcal{P} \mathcal{T}_{Y}$; we will refer to this incomplete action as the augmented action of the original partial action. The expansion method similarly results in the construction of an incomplete action.

We begin the paper with a brief overview of weakly left $E$-ample semigroups (Section 2). In Section 3, we give appropriate definitions of partial actions and premorphisms in the weakly left $E$-ample case, and connect these with the existing definitions in the inverse case.

Section 4 introduces the generalized Szendrei expansion of a weakly left $E$-ample semigroup, adapted very slightly from [10]. This is a vital ingredient in the 'expansion' method, which we describe in Section 5. We note that weakly left $E$-ample semigroups come equipped with a natural partial order. Gomes observed in [10, p. 397] that if $\phi: T \rightarrow S$ is a $(2,1)$-morphism onto $S$, where $S$ and $T$ are weakly left ample semigroups such that for any $s \in S, s \phi^{-1}$ contains a maximum element $s \phi^{*}$, then $\phi^{*}: S \rightarrow T$ is a weak premorphism; this easily extends to the case where $E$ may not be the whole set of idempotents. If, in addition, $\phi^{*}$ is strong, then $\phi$ is called an FA-morphism from $T$ onto $S$; the reader should be aware that, in view of Lemma 3.10, every strong premorphism is order-preserving, as insisted upon in the definition of an $F A$-morphism. We show that any strong premorphism $\theta: S \rightarrow T$, where $S$ and $T$ are weakly left $E$ - $(F$-)ample, respectively, is associated with an $F A$-morphism $\phi: A \rightarrow S$, for some weakly left $G$-ample semigroup $A$, and a $(2,1)$ morphism $\rho: A \rightarrow T$ such that $\phi^{*} \rho=\theta$. Our results in Section 5 are then easily seen to be equivalent to those of [10]. By specializing to the inverse case, we recover the expansion result of [19, Proposition 6.20].

Section 6 details the construction of the 'augmented action' of a given partial action. Again, we can specialize to partial actions of inverse semigroups. Specifically, we show that $\theta: S \rightarrow \mathcal{I}_{X}$ is an inverse semigroup premorphism if, and only if, there exist $Y \supseteq X$ and a morphism $\varphi: S \rightarrow \mathcal{I}_{Y}$ such that $s \theta=\iota(s \varphi) \iota^{-1}$, for all $s \in S$. 


\section{Weakly left $E$-ample semigroups}

We give here a very brief overview of weakly left $E$-ample semigroups. Further details can be found in the summary [12 $]^{1}$ or [13, Section 1], written for monoids, from which most of this material is taken. Another useful source for the reader wanting to see how weakly left $E$-ample semigroups are related to the function systems of Schweizer and Sklar [29] and the twisted $R C$-semigroups of Jackson and Stokes [15] is the survey article [16], which itself draws much of its material from the comprehensive paper by Schein [25]. We remark that the ' $E$ ' in 'weakly left $E$-ample' is both a generic term and used to refer to a particular set of idempotents. We use the letter ' $E$ ' where there is no danger of ambiguity; otherwise we employ ' $F$ ', ' $G$ ', and so on.

Weakly left $E$-ample semigroups arise very naturally from partial transformation monoids in the same way that inverse semigroups arise from symmetric inverse monoids. Let $X$ be a nonempty set. It is well known that the partial transformation monoid $\mathcal{P} \mathcal{T}_{X}$ contains a semilattice of idempotents

$$
E_{X}=\left\{I_{A}: A \subseteq X\right\}
$$

where $I_{A}$ is the identity map on $A$. In other words, $E_{X}$ is the set of partial identities in $\mathcal{P} \mathcal{T}_{X}$. We now define a unary operation ${ }^{+}$on $\mathcal{P} \mathcal{T}_{X}$ by $\alpha^{+}=I_{\mathrm{dom} \alpha}$, for each $\alpha \in \mathcal{P} \mathcal{T}_{X}$. Let $S$ be a subsemigroup of $\mathcal{P} \mathcal{T}_{X}$ and let $E$ be the set

$$
E=\left\{I_{Z} \in E_{X}: Z=\operatorname{dom} \alpha \text {, for some } \alpha \in S\right\}
$$

If $S$ is closed under ${ }^{+}$, that is, if $E \subseteq S$, then we call $S$ a weakly left $E$-ample semigroup. Such a subsemigroup can be regarded as a $(2,1)$-subalgebra of $\mathcal{P} \mathcal{T}_{X}$. Since $\mathcal{P} \mathcal{T}_{X}$ itself is closed under ${ }^{+}$, certainly $\mathcal{P} \mathcal{T}_{X}$ is weakly left $E_{X}$-ample.

Weakly left $E$-ample semigroups also have an abstract characterization of which we will make extensive use. Let $S$ be a semigroup and suppose that $E \subseteq E(S)$ is a subsemilattice of $S$. We define the (equivalence) relation $\widetilde{\mathcal{R}}_{E}$ on $S$ by the rule that

$$
a \widetilde{\mathcal{R}}_{E} b \Longleftrightarrow \forall e \in E[e a=a \Leftrightarrow e b=b]
$$

for $a, b \in S$. Thus, two elements $a, b$ are $\widetilde{\mathcal{R}}_{E}$-related if, and only if, they have the same left identities in $E$. In view of Theorem 2.2 below, there is no danger of ambiguity in making the following definition.

DEFINITION 2.1. A semigroup $S$ with subsemilattice $E \subseteq E(S)$ is weakly left E-ample if:

\footnotetext{
${ }^{1}$ We note that whilst all of the material of this section may be found in a variety of published papers $([5,6,11,15,18]$, for instance), the set of notes [12] is the only place where the relevant definitions and results have been collated into a single resource; for this reason, [12] is a useful reference for our purposes.
} 
(1) every element $a$ is $\widetilde{\mathcal{R}}_{E}$-related to a (necessarily unique) idempotent in $E$, denoted $a^{+}$;

(2) $\widetilde{\mathcal{R}}_{E}$ is a left congruence;

(3) for all $a \in S$ and all $e \in E$, ae $=(a e)^{+} a$.

Thus, in a weakly left $E$-ample semigroup $S, a \widetilde{\mathcal{R}}_{E} b$ if, and only if, $a^{+}=b^{+}$. The idempotent $a^{+}$is a left identity for $a$. It is also clear that if $e \in E$, then $e^{+}=e$. The identity in condition (3) will be referred to throughout as the 'left ample identity'. We note the following simpler condition for an element $a \in S$ to be $\widetilde{\mathcal{R}}_{E}$-related to an idempotent $e \in E$ :

$$
a \widetilde{\mathcal{R}}_{E} e \Longleftrightarrow e a=a \text { and } \forall f \in E[f a=a \Rightarrow f e=e] .
$$

The following theorem, which first appears in [31], connects the two approaches to weakly left $E$-ample semigroups.

THEOREM 2.2. [12, Theorem 5.2] Let $S$ be a weakly left E-ample semigroup, regarded as an algebra of type $(2,1)$, for some $E \subseteq E(S)$. Then the mapping $\phi: S \rightarrow$ $\mathcal{P} \mathcal{T}_{S}$ given by $\phi \phi=\rho_{s}$, where

$$
\operatorname{dom} \rho_{s}=S s^{+} \quad \text { and } \quad x \rho_{s}=x s, \quad \forall x \in \operatorname{dom} \rho_{s},
$$

is a representation of $S$ as a (2,1)-subalgebra of $\mathcal{P} \mathcal{T}_{S}$.

Thus weakly left $E$-ample semigroups are, up to isomorphism, precisely (2,1)subalgebras of partial transformation monoids.

For any semigroup $S$, if $E=E(S)$, then we denote $\widetilde{\mathcal{R}}_{E}$ by $\widetilde{\mathcal{R}}$. Note that $\widetilde{\mathcal{R}} \subseteq \widetilde{\mathcal{R}}_{E}$, for any $E$. If $S$ is weakly left $E$-ample with $E=E(S)$, then we call $S$ simply weakly left ample.

It is easy to see that, in a regular semigroup, $\mathcal{R}=\widetilde{\mathcal{R}}$. It follows that weakly left ample semigroups generalize inverse semigroups, since every inverse semigroup is weakly left ample with $a^{+}=a a^{-1}$. Note that every monoid is weakly left $\{1\}$-ample, with $a^{+}=1$, for all elements $a$. A unipotent monoid is therefore weakly left ample. Weakly left $E$-ample semigroups also generalize the left ample (formerly, left type-A) semigroups of Fountain [5, 6].

Let $S$ be a weakly left $E$-ample semigroup. For the purposes of Section 6, we observe that if we adjoin an identity 1 to $S$, then $S^{1}$ is a weakly left $E^{1}$-ample monoid.

We note a useful identity involving ${ }^{+}$which follows easily from the fact that $\widetilde{\mathcal{R}}_{E}$ is a left congruence.

LEMMA 2.3. Let $S$ be a weakly left E-ample semigroup, for some $E \subseteq E(S)$, and let $s, t \in S$. Then $(s t)^{+}=\left(s t^{+}\right)^{+}$.

The partial transformation monoid $\mathcal{P} \mathcal{T}_{X}$ possesses an obvious natural partial order (that is, a partial order which is compatible with multiplication and which restricts to the usual partial order on idempotents):

$$
\alpha \leq \beta \Longleftrightarrow \alpha=\left.\beta\right|_{\text {dom } \alpha} .
$$


Note that $\mathcal{T}_{X}$, the full transformation monoid on $X$, is a submonoid of $\mathcal{P} \mathcal{T}_{X}$. Since the elements of $\mathcal{T}_{X}$ are defined on the whole of $X$, the ordering becomes trivial. In the abstract characterization of a weakly left $E$-ample semigroup $S$, the ordering of (2.2) becomes the following natural partial order

$$
a \leq b \Longleftrightarrow a=e b
$$

for some idempotent $e \in E$. Equivalently,

$$
a \leq b \Longleftrightarrow a=a^{+} b
$$

This equivalence is justified in [13, Section 1].

LEMMA 2.4. Let $S$ be a weakly left E-ample semigroup with partial order $\leq$. If $s \in S$ and $e \in E$, then $s e \leq s$.

PROOF. Applying the left ample identity, we have $s e=(s e)^{+} s \leq s$, by (2.3).

We observed earlier that $a^{+}$is a left identity for $a$. We can now say a little more: $a^{+}$is the least left identity for $a$, with respect to $\leq$. This is a consequence of (2.1), together with the definition of $\leq$. The next result now follows.

LEMMA 2.5 [6, Proposition 1.6]. Let $S$ be a weakly left E-ample semigroup with partial order $\leq$, and let $s, t \in S$. Then $(s t)^{+} \leq s^{+}$.

\section{Partial actions and premorphisms}

We begin by summarizing some existing definitions for the actions and partial actions of monoids; we will shortly adapt these to the case of a weakly left $E$-ample semigroup. First, recall the definition of the (global) action of a monoid $M$ on a set $X$ :

Definition 3.1. A monoid $M$ acts (globally) on a set $X$ (on the right) if there is a mapping $X \times M \rightarrow X$, given by $(x, s) \mapsto x \cdot s$, and such that:

(1) $x \cdot 1=x$, for all $x \in X$;

(2) $(x \cdot s) \cdot t=x \cdot s t$, for all $x \in X$, for all $s, t \in M$.

The concept of a monoid action generalizes to that of a partial monoid action, in which the action $x \cdot s$ is not necessarily defined for all pairs $(x, s) \in X \times M$. We will write ' $\exists x \cdot s$ ' to mean 'the action of $s \in M$ on $x \in X$ is defined'. As commented in the Introduction, there exists more than one definition of a 'partial monoid action' in the literature. We have, for example, the definition used in [23] for the 'partial actions' of inverse monoids. Since this is not the definition of 'partial action' which we will ultimately adopt, we record it under the name of incomplete action.

Definition 3.2. The incomplete action of a monoid $M$ on a set $X$ (on the right) is a partial mapping $X \times M \rightarrow X$, given by $(x, s) \mapsto x \cdot s$, and such that: 
(PA1) $\exists x \cdot 1$ and $x \cdot 1=x$, for all $x \in X$;

(PA2*) $\exists x \cdot s$ and $\exists(x \cdot s) \cdot t \Leftrightarrow \exists x \cdot s t$, in which case $x \cdot s t=(x \cdot s) \cdot t$.

Incomplete actions were discussed briefly in [13, p. 297] (though not under this name); it was observed that it is very easy to construct a global action from an incomplete action: we simply adjoin an extra symbol, say 0 , to $X$ and then define all previously undefined actions to be equal to 0 , thereby obtaining the global action of $M$ on $X \cup\{0\}$. The concept of an incomplete action will nevertheless prove very useful later on.

The notion of partial action which we will adopt is adapted from that of [13], in which can be found two further notions of partial monoid action. The first of these, a weak partial action [13, Definition 2.2], is obtained from the partial group action of Kellendonk and Lawson [17, p. 87] in a naive way simply by deleting all reference to inverses. However, it is the second, slightly stronger, notion of partial monoid action, a strong partial action [13, Definition 2.4], which emerges as being natural. It is worth noting that the class of weak partial actions properly contains the class of strong partial actions (see [13, Example 2.11]), which, in turn, properly contains the class of incomplete actions (see Examples 2 and 3).

Each of the definitions of [13] can, of course, be applied to any monoid; however, in the particular case of a weakly left $E$-ample monoid, any results obtained by using these definitions (in particular, the results of [13]) will not, in general, respect the ${ }^{+}$ operation. Therefore, for weakly left $E$-ample monoids, we choose to augment both definitions of partial action (as well as the definition of an incomplete action) with an additional axiom which reflects the presence of ${ }^{+}$.

In the inverse case, the existing work on partial actions concerns semigroups, rather than monoids. Also, as will be seen in Sections 4 and 5, we no longer need the presence of an identity in order for the 'expansion' method to be applied. For these reasons, we deal with partial semigroup actions, rather than partial monoid actions. The adaptation to the semigroup case is effected simply by deleting the 'identity axiom' - axiom (PA1) in Definition 3.2; axiom (1) in Definition 3.1. In Section 6, we will find it necessary temporarily to reinstate axiom (PA1) for strong partial actions; we will then refer to strong unitary partial actions. In the interests of consistency, we retain the numbering of [13].

Definition 3.3 (Weak partial action). A weakly left $E$-ample semigroup $S$ acts partially (and 'weakly') on a set $X$ (on the right) if there is a partial mapping $X \times S \rightarrow X$, given by $(x, s) \mapsto x \cdot s$, and such that:

$\left(\mathrm{PA} 2^{\prime}\right) \exists x \cdot s$ and $\exists(x \cdot s) \cdot t \Rightarrow \exists x \cdot s t$, in which case $x \cdot s t=(x \cdot s) \cdot t$;

(PA3) $\exists x \cdot s \Rightarrow \exists x \cdot s^{+}$and $x \cdot s^{+}=x$.

Definition 3.4 (Strong partial action). A weakly left $E$-ample semigroup $S$ acts partially and 'strongly' on a set $X$ (on the right) if there is a partial mapping $X \times S \rightarrow X$, given by $(x, s) \mapsto x \cdot s$, and such that: 
(PA2) $\exists x \cdot s \Rightarrow[\exists(x \cdot s) \cdot t \Leftrightarrow \exists x \cdot s t]$, in which case $x \cdot s t=(x \cdot s) \cdot t$;

(PA3) $\exists x \cdot s \Rightarrow \exists x \cdot s^{+}$and $x \cdot s^{+}=x$.

DEFINITION 3.5 (Incomplete action). The incomplete action of a weakly left $E$ ample semigroup $S$ on a set $X$ (on the right) is a partial mapping $X \times S \rightarrow X$, given by $(x, s) \mapsto x \cdot s$, and such that:

$\left(\mathrm{PA} 2^{*}\right) \exists x \cdot s$ and $\exists(x \cdot s) \cdot t \Leftrightarrow \exists x \cdot s t$, in which case $x \cdot s t=(x \cdot s) \cdot t$;

$\left(\mathrm{PA}^{*}\right) \exists x \cdot s \Leftrightarrow \exists x \cdot s^{+}$and $x \cdot s^{+}=x$.

Notice that by (PA3), for any $e \in E$, if $\exists x \cdot e$, then $x \cdot e=x$. The reasons for including the stronger axiom (PA3*) in Definition 3.5 will be explained shortly.

Suppose that we now let $S$ be an arbitrary monoid, regarded as being weakly left $\{1\}$-ample with $s^{+}=1$, for all $s \in S$. Then condition (PA3) holds automatically and Definitions 3.3 and 3.4 (with (PA1) restored) reduce to [13, Definitions 2.2 and 2.4], respectively. In what follows, 'weak partial action' and 'strong partial action' will mean the partial actions of Definitions 3.3 and 3.4 of the present paper, respectively.

Returning to the general case, we present two examples of partial actions of weakly left $E$-ample semigroups: one of a weak partial action which is not strong, and one of a strong partial action which is not an incomplete action.

EXAMPLE 1. Let $S$ be a weakly left $E$-ample semigroup and let $I$ be a nonempty set. We denote by $Q$ the $I \times I$ identity matrix and consider the Rees matrix semigroup $\mathcal{M}:=\mathcal{M}^{0}(S ; I, I ; Q)$. Multiplication in $\mathcal{M}$ is given by

$$
0(i, s, j)=0=00=(i, s, j) 0,
$$

and

$$
(i, s, j)(k, t, l)= \begin{cases}(i, s t, l) & \text { if } j=k \\ 0 & \text { if } j \neq k\end{cases}
$$

The idempotents of $\mathcal{M}$ are those elements of the form $(i, e, i)$, for $e \in E(S)$, together with 0 . In [14, Example 2.7.3], it is shown that $\mathcal{M}$ is weakly left $\mathscr{E}$-ample with $0^{+}=0$ and $(i, s, j)^{+}=\left(i, s^{+}, i\right)$, where

$$
\mathscr{E}=\{(i, e, i) \in E(\mathcal{M}): e \in E\} \cup\{0\} .
$$

We define a partial action of $\mathcal{M}$ on $I$ by:

$$
\nexists a \cdot 0 \text { for any } a \in I,
$$

and

$$
\exists a \cdot(i, s, j) \Longleftrightarrow a=i \quad \text { and } \quad s \in E \quad \text { in which case, } a \cdot(i, s, j)=j .
$$

We verify that this is a weak partial action:

(PA2') Suppose that $\exists a \cdot(i, s, j)$ and $\exists(a \cdot(i, s, j)) \cdot(k, t, l)$, so that $a=i, j=k$ and $s, t \in E$. Then $(i, s, j)(k, t, l)=(i, s t, l)$ and $s t \in E$, so $\exists a \cdot(i, s, j)(k, t, l)$ and $a \cdot(i, s, j)(k, t, l)=(a \cdot(i, s, j)) \cdot(k, t, l)$. 
(PA3) If $\exists a \cdot(i, s, j)$, then $a=i$ and $s=s^{+} \in E$, so that $\exists a \cdot(i, s, j)^{+}$and $a \cdot(i, s, j)^{+}=a \cdot\left(i, s^{+}, i\right)=i=a$.

We now show that the full condition (PA2) does not hold. Suppose that $\exists a \cdot(i, s, j)$ and that $\exists a \cdot(i, s t, l)$. Then $a=i$ and $s, s t \in E$. However, in general, there is no guarantee that we will have $t \in E$ : we can choose $S$ to be a weakly left $E$-ample semigroup in which $E$ does not form a unitary subset. Indeed, it is easy to see that $E_{X}$ is not right unitary in $\mathcal{P} \mathcal{T}_{X}$ for any $X$ with $|X| \geq 2$. Therefore, in general, we cannot deduce that $\exists(a \cdot(i, s, j)) \cdot(k, t, l)$. We have shown that this is a weak partial action which is not strong.

EXAMPLE 2. Let $S$ be a weakly left $E$-ample semigroup with subset $T$. We define a strong partial action of $S$ on $T$ via the formula

$$
\exists x \cdot s \Longleftrightarrow x \in T s^{+} \text {and } \quad x s \in T \text { in which case } x \cdot s=x s .
$$

We verify that this is indeed a strong partial action:

(PA2) Suppose that $\exists x \cdot s$ and $\exists(x \cdot s) \cdot t$. We therefore have $x \in T s^{+}$and $x s \in$ $T t^{+}$. Consequently, $x s^{+}=x$ and $x s t^{+}=x s$. Then

$$
\begin{aligned}
x & =x s^{+}=\left(x s^{+}\right)^{+} x \quad \text { by the left ample identity, } \\
& =(x s)^{+} x \quad \text { by Lemma } 2.3, \\
& =\left(x s t^{+}\right)^{+} x=(x s t)^{+} x \quad \text { since } x s=x s t^{+} \\
& =\left(x(s t)^{+}\right)^{+} x=x(s t)^{+} \in T(s t)^{+} .
\end{aligned}
$$

Also, $x s t \in T$, so $\exists x \cdot s t$ and $x \cdot s t=(x \cdot s) \cdot t$.

Conversely, suppose that $\exists x \cdot s$ and $\exists x \cdot s t$. We have $x=x(s t)^{+}$and therefore

$$
x s=x(s t)^{+} s=x\left(s t^{+}\right)^{+} s=x s t^{+} \in T t^{+},
$$

since $x s \in T$. Hence $\exists(x \cdot s) \cdot t$.

(PA3) If $\exists x \cdot s$, then $x \in T s^{+}=T\left(s^{+}\right)^{+}$. Also, $x=x s^{+}$, as before, so $x s^{+} \in T$. Therefore, $\exists x \cdot s^{+}$, with $x \cdot s^{+}=x$.

Note that this is an example of a strong partial action which is not, in general, an incomplete action. To see this, let $S$ be the semigroup of [11, Example 2.6]:

$$
S=\left\{\alpha, \beta, \alpha^{+}, \tau, \tau^{+}\right\} \subseteq \mathcal{I}_{\{1,2,3,4,5\}},
$$

where

$$
\alpha=\left(\begin{array}{ccccc}
1 & 2 & 3 & 4 & 5 \\
2 & \times & \times & 5 & 4
\end{array}\right), \quad \beta=\left(\begin{array}{ccccc}
1 & 2 & 3 & 4 & 5 \\
3 & \times & \times & 5 & 4
\end{array}\right)
$$

and

$$
\tau=\left(\begin{array}{ccccc}
1 & 2 & 3 & 4 & 5 \\
\times & \times & \times & 5 & 4
\end{array}\right),
$$

and $\mathcal{I}_{\{1,2,3,4,5\}}$ is the symmetric inverse monoid on $\{1,2,3,4,5\}$. Then $S$ is a (weakly) left ample semigroup with multiplication table 


\begin{tabular}{c|ccccc} 
& $\alpha^{+}$ & $\alpha$ & $\beta$ & $\tau^{+}$ & $\tau$ \\
\hline$\alpha^{+}$ & $\alpha^{+}$ & $\alpha$ & $\beta$ & $\tau^{+}$ & $\tau$ \\
$\alpha$ & $\tau$ & $\tau^{+}$ & $\tau^{+}$ & $\tau$ & $\tau^{+}$ \\
$\beta$ & $\tau$ & $\tau^{+}$ & $\tau^{+}$ & $\tau$ & $\tau^{+}$ \\
$\tau^{+}$ & $\tau^{+}$ & $\tau$ & $\tau$ & $\tau^{+}$ & $\tau$ \\
$\tau$ & $\tau$ & $\tau^{+}$ & $\tau^{+}$ & $\tau$ & $\tau^{+}$
\end{tabular}

The $\widetilde{\mathcal{R}}$-classes of $S$ are $\left\{\alpha^{+}, \alpha, \beta\right\}$ and $\left\{\tau^{+}, \tau\right\}$.

We put $T=S \backslash E(S)=\{\alpha, \beta, \tau\}$. Observe that $\exists \tau \cdot \tau^{+}$, since $\tau=\alpha \tau^{+} \in T \tau^{+}$and $\tau \tau^{+}=\tau \in T$. We see from the multiplication table that $\alpha^{2}=\tau^{+}$, so $\exists \tau \cdot \alpha^{2}$. However, the action $\tau \cdot \alpha$ is not defined, since $\tau \alpha=\tau^{+} \notin T$. Therefore this is not an incomplete action.

The global action of a semigroup $S$ on a set $X$ is, of course, equivalent to a morphism $\varphi: S \rightarrow \mathcal{T}_{X}$, where $\mathcal{T}_{X}$ is the full transformation monoid on $X$; for each $s \in S, s \varphi$ is the mapping $x \mapsto x \cdot s$. Similarly, the incomplete action of $S$ is equivalent to a morphism $\psi: S \rightarrow \mathcal{P} \mathcal{T}_{X}$; in this case, $x \in \operatorname{dom} s \psi$ if, and only if, $\exists x \cdot s$. The mapping to which a partial action is equivalent is a premorphism; a weak partial monoid action (in the original sense of [13]) is equivalent to a (weak) premorphism, whilst a strong partial monoid action is equivalent to a strong premorphism. Each of these concepts was adapted (in [13, Section 2]) from the ' $\wedge$-prehomomorphism' of McAlister and Reilly [21] and the 'unital group premorphism' of Kellendonk and Lawson [17]. As with partial actions, every strong premorphism is weak, but not every weak premorphism is strong.

We have already adapted our concept of partial action to take account of the ${ }^{+}$ operation, so we must now adapt our concept of premorphism. We naturally do this by adding an extra axiom to reflect the presence of ${ }^{+}$. Again, we move to the semigroup case by deleting the 'identity axiom' of [13]: $1 \theta=1$.

DEFINITION 3.6 (Weak premorphism). Let $S$ and $T$ be semigroups, with $S$ weakly left $E$-ample and $T$ weakly left $F$-ample, for some $E \subseteq E(S)$ and $F \subseteq E(T)$. Then $\theta: S \rightarrow T$ is a weak premorphism if:

$\left(\mathrm{PM} 2^{\prime}\right)(s \theta)(t \theta) \leq(s t) \theta$;

(PM3) $(s \theta)^{+} \leq s^{+} \theta$,

where $\leq$ is the natural partial order in $T$, as defined by (2.3).

Definition 3.7 (Strong premorphism). Let $S$ and $T$ be as in Definition 3.6. Then $\theta: S \rightarrow T$ is a strong premorphism if:

(PM2) $(s \theta)(t \theta)=(s \theta)^{+}(s t) \theta$;

(PM3) $(s \theta)^{+} \leq s^{+} \theta$.

In Section 6, we will find it necessary to reinstate the identity axiom $1 \theta=1$ for strong premorphisms. In the interests of clarity, we will then refer to strong unitary premorphisms. 
Observe that if $S$ is an arbitrary monoid, again regarded as being weakly left $\{1\}$-ample, then condition (PM3) becomes $(s \theta)^{+} \leq 1$, by (PM1). This condition holds automatically, by definition of $\leq$. Definitions 3.6 and 3.7 therefore reduce to [13, Definitions 2.7 and 2.9], respectively. In what follows, 'weak premorphism' and 'strong premorphism' will mean the mappings of Definitions 3.6 and 3.7 of the present paper, respectively.

Returning once more to the general case, we make the easy observation that, thanks to axiom (PA3*), the incomplete action of a weakly left $E$-ample semigroup $S$ on a set $X$ is equivalent to a $(2,1)$-morphism $\varphi: S \rightarrow \mathcal{P} \mathcal{T}_{X}$. We also extend the results of [13, Section 2].

Proposition 3.8. A weak partial action of a weakly left E-ample semigroup $S$ on a set $X$ is equivalent to a weak premorphism $\theta: S \rightarrow \mathcal{P} \mathcal{T}_{X}$.

PROOF. As for incomplete actions, if $S$ acts (weakly and) partially on $X$, then we define $\theta: S \rightarrow \mathcal{P} \mathcal{T}_{X}$ by the rule that for any $s \in S, x \in \operatorname{dom} s \theta$ if, and only if, $\exists x \cdot s$, and for $x \in \operatorname{dom} s \theta, x(s \theta)=x \cdot s$. On the other hand, if $\theta: S \rightarrow \mathcal{P} \mathcal{T}_{X}$ is a weak premorphism, we can define a partial map $X \times S \rightarrow X,(x, s) \rightarrow x \cdot s=x(s \theta)$.

From [13, Proposition 2.8], each of conditions (PA2') and (PM2') implies the other. It only remains to show that each of (PA3) and (PM3) implies the other.

First, suppose that the weak partial action satisfies (PA3) and let $x \in \operatorname{dom} s \theta$. Then $x \in \operatorname{dom}(s \theta)^{+}$and $x(s \theta)^{+}=x$, since $(s \theta)^{+}=I_{\mathrm{dom} s} \theta$. Now, since $x \in \operatorname{dom} s \theta$, we have $\exists x \cdot s$, hence $\exists x \cdot s^{+}$with $x \cdot s^{+}=x$, by (PA3). In terms of $\theta$, this becomes $x \in \operatorname{dom} s^{+} \theta$ and $x\left(s^{+} \theta\right)=x$. Thus $(s \theta)^{+} \leq s^{+} \theta$.

Conversely, let $\theta: S \rightarrow \mathcal{P} \mathcal{T}_{X}$ be a weak premorphism which satisfies (PM3). Suppose that $\exists x \cdot s$, that is, $x \in \operatorname{dom} s \theta$. Then $x \in \operatorname{dom}(s \theta)^{+}$and $x(s \theta)^{+}=x$. Now, $x \in \operatorname{dom} s^{+} \theta$ and $x\left(s^{+} \theta\right)=x$, by (PM3), so $\exists x \cdot s^{+}$and $x \cdot s^{+}=x$.

\section{PROPOSITION 3.9. A strong partial action is equivalent to a strong premorphism.}

PROOF. This follows by combining the proof of Proposition 3.8 with parts of that of [13, Proposition 2.10].

Note that in the monoid case a strong unitary premorphism is equivalent to a strong unitary partial action.

EXAMPLE 3. Let $S$ be a weakly left ample semigroup and let $X$ be an $\widetilde{\mathcal{R}}$-class of $S$. We define a mapping $\theta: S \rightarrow \mathcal{P} \mathcal{T}_{X}$ by:

$$
\operatorname{dom} s \theta=\{x \in X: x s=x\} \quad \text { and } \quad x(s \theta)=x s .
$$

We show that $\theta$ is a strong premorphism by switching to the equivalent partial action: $\exists x \cdot s$ if, and only if, $x s=x$, in which case, $x \cdot s=x s=x$.

(PA2) Suppose that $\exists x \cdot s$ and $\exists(x \cdot s) \cdot t$, that is, $x s=x$ and $x s t=x s$. Clearly, $x s t=x$, so $\exists x \cdot s t$ and $(x \cdot s) \cdot t=x \cdot s t$. The converse is similar. 
(PA3) Suppose that $\exists x \cdot s$. Then $x s=x$. By Lemma 2.3, $\left(x s^{+}\right)^{+}=x^{+}$, so $x=x^{+} x=\left(x s^{+}\right)^{+} x=x s^{+}$, hence $\exists x \cdot s^{+}$and $x \cdot s^{+}=x s^{+}=x$.

Note that this is another example of a strong partial action which is not an incomplete action (alternatively, $\theta$ is a strong premorphism which is not a morphism). To see this, let us reuse the semigroup of Example 2 with $X=\left\{\tau^{+}, \tau\right\}$. We see that $\tau \tau^{+}=\tau$, so $\exists \tau \cdot \tau^{+}$. From the multiplication table for $S$, we have $\tau^{+}=\alpha^{2}$, so $\exists \tau \cdot \alpha^{2}$. However, $\tau \alpha \neq \tau$, so the action $\tau \cdot \alpha$ is not defined. Therefore this is not an incomplete action.

We note some useful properties of strong premorphisms.

LEMMA 3.10. Let $\theta: S \rightarrow T$ be a strong premorphism between the weakly left $E$ and $F$-ample semigroups $S$ and $T$. Then $\theta$ has the following properties:

(1) $e \in E(S) \Rightarrow e \theta \in E(T)$;

(2) $e \in E \Rightarrow e \theta \in F$;

(3) $e, f \in E, e \leq f \Rightarrow e \theta \leq f \theta$;

(4) $u, v \in S, u \leq v \Rightarrow u \theta \leq v \theta$.

Proof. (1) Suppose that $e \in E(S)$. Then $(e \theta)(e \theta)=(e \theta)^{+}(e e) \theta=e \theta$, so $e \theta \in$ $E(T)$.

(2) Suppose that $e \in E$. By (1), we know that $e \theta \in E(T)$. Since $e \in E$, then $(e \theta)^{+} \leq e^{+} \theta=e \theta$. By definition of $\leq,(e \theta)^{+}=(e \theta)^{+}(e \theta)=e \theta$, so $e \theta \in F$.

(3) Let $e, f \in E$ be such that $e \leq f$. Then $e=e f$, so $e \theta=(e f) \theta=(e \theta)(e f) \theta$ $=(e \theta)^{+}(e f) \theta=(e \theta)(f \theta)$. Thus $e \theta \leq f \theta$.

(4) Let $u, v \in S$ be such that $u \leq v$. Then $u=e v$, for some $e \in E$. Therefore, $u \theta=(e v) \theta \Rightarrow(e \theta)(u \theta)=(e \theta)(e v) \theta=(e \theta)^{+}(e v) \theta=(e \theta)(v \theta)$. But $u^{+} \leq e$, by Lemma 2.5. Then $(u \theta)^{+} \leq u^{+} \theta \leq e \theta$, by (3). We can therefore multiply $(e \theta)(u \theta)$ $=(e \theta)(v \theta)$ on the left by $(u \theta)^{+}$to obtain $u \theta=(u \theta)^{+}(v \theta)$. Hence, $u \theta \leq v \theta$.

It is worth emphasizing that a strong premorphism is perforce order-preserving, so this does not need to be given as a dedicated condition.

Suppose that $\theta: S \rightarrow T$ is a strong premorphism of weakly left $E$-ample semigroups $S$ and $T$. We adjoin identities to both $S$ and $T$ and define a new mapping $\theta^{\prime}: S^{1} \rightarrow T^{1}$ as follows:

$$
1 \theta^{\prime}=1 \quad \text { and } \quad s \theta^{\prime}=s \theta \quad \text { for all } s \in S .
$$

The following result is easy to verify.

LEMMA 3.11. The mapping $\theta^{\prime}$ is a strong unitary premorphism.

In particular, if $\theta: S \rightarrow \mathcal{P} \mathcal{T}_{X}$ is a strong premorphism, then $\theta^{\prime}: S^{1} \rightarrow \mathcal{P} \mathcal{T}_{X}$ is a strong unitary premorphism with $1 \theta^{\prime}=I_{X}$.

We end this section with a consideration of the inverse case. Recall from [19] that a mapping $\theta: S \rightarrow T$ between inverse semigroups $S$ and $T$ is called a dual prehomomorphism if $(s \theta)(t \theta) \leq(s t) \theta$ and $(s \theta)^{-1}=s^{-1} \theta$, for all $s, t \in S$. In order to 
bring this into line with our terminology, we will refer to such a mapping as an inverse semigroup premorphism. In [19], the partial action of an inverse semigroup $S$ on a set $X$ is defined to be an order-preserving inverse semigroup premorphism $S \rightarrow \mathcal{I}_{X}$, where $\mathcal{I}_{X}$ is the symmetric inverse monoid on $X$.

LEMMA 3.12. Let $\theta: S \rightarrow T$ be a mapping between inverse semigroups $S$ and $T$. Then $\theta$ is an order-preserving inverse semigroup premorphism if, and only if, it is a strong premorphism of weakly left ample semigroups.

PROOF. Suppose first that $\theta$ is an order-preserving inverse semigroup premorphism. Then for any $s, t \in S$,

$$
\begin{aligned}
(s \theta)^{+}(s t) \theta & =(s \theta)(s \theta)^{-1}(s t) \theta \\
& =(s \theta)\left(s^{-1} \theta\right)(s t) \theta \\
& \leq(s \theta)\left(s^{-1} s t\right) \theta \\
& \leq(s \theta)(t \theta)
\end{aligned}
$$

since $s^{-1} s t \leq t$ and $\theta$ is order-preserving. It follows that $(s \theta)(t \theta)=(s \theta)^{+}(s t) \theta$. Further,

$$
(s \theta)^{+}=(s \theta)(s \theta)^{-1}=(s \theta)\left(s^{-1} \theta\right) \leq\left(s s^{-1}\right) \theta=s^{+} \theta,
$$

so that $\theta$ is a strong premorphism of weakly left ample semigroups.

Conversely, suppose that $\theta$ is a strong premorphism. By Lemma 3.10, $\theta$ is orderpreserving. For any $s \in S$, we have that

$$
(s \theta)\left(s^{-1} \theta\right)(s \theta)=(s \theta)^{+}\left(s s^{-1}\right) \theta(s \theta)=(s \theta)^{+}\left(s^{+} \theta\right)(s \theta)=(s \theta)^{+}(s \theta)=s \theta,
$$

whence it follows that $(s \theta)^{-1}=s^{-1} \theta$, as required.

A partial action of an inverse semigroup is defined to be one-one, in the sense that the image of the equivalent strong premorphism contains only one-one maps. In fact, this does not need to be specified, as we now show.

LEMMA 3.13. Let $S$ be an inverse semigroup and let $\theta: S \rightarrow \mathcal{P} \mathcal{T}_{X}$ be a strong premorphism. Then $\operatorname{im} \theta \subseteq \mathcal{I}_{X}$.

PRoOf. Let $s \in S$ and $x, y \in \operatorname{dom} s \theta$, and suppose that $x(s \theta)=y(s \theta)$. Then $x, y \in$ $\operatorname{dom} s \theta=\operatorname{dom}(s \theta)^{+}$. Now,

$$
(s \theta)\left(s^{-1} \theta\right)=(s \theta)^{+}\left(s s^{-1}\right) \theta=(s \theta)^{+}\left(s^{+} \theta\right)=(s \theta)^{+} .
$$

Therefore, $x, y \in \operatorname{dom}(s \theta)\left(s^{-1} \theta\right)$ and $x(s \theta), y(s \theta) \in \operatorname{dom} s^{-1} \theta$. Hence,

$$
\begin{aligned}
x(s \theta)=y(s \theta) & \Rightarrow x(s \theta)\left(s^{-1} \theta\right)=y(s \theta)\left(s^{-1} \theta\right) \\
& \Rightarrow x(s \theta)^{+}=y(s \theta)^{+},
\end{aligned}
$$

whence $x=y$, as required.

Thus, the strong partial actions of an inverse semigroup $S$ (in the sense of Definition 3.4) are precisely its partial actions in the sense of [19]. 


\section{The Szendrei expansion of a weakly left $E$-ample semigroup}

Our aim is to obtain analogues of results from [13] concerning both the 'expansion' and 'globalization' of partial actions of weakly left $E$-ample semigroups. We begin with the 'expansion' method. Given the partial action of a weakly left $E$-ample semigroup $S$ on a set $X$, our goal is to construct the incomplete action of some new weakly left $E^{\prime}$-ample semigroup $S^{\prime}$ on $X$. We do this in much the same way as in [13]: by taking an expansion of $S$.

Semigroup expansions were first introduced by Birget and Rhodes [3] and are formally defined as follows.

Definition 4.1 [3, p. 241]. An expansion is a functor $F$ from one category of semigroups to a larger one such that there exists a natural transformation $v$ from $F$ to the identity functor, with each arrow $v_{S}$ surjective.

Since an expansion is a functor, we should not only specify its effect on the objects of a category but also its effect on the arrows. However, the 'objects' part is the only aspect of an expansion that we will need in connection with actions and partial actions. We will therefore omit all reference to an expansion's effect on arrows.

In [13] (as well as in [17] for partial group actions), the Szendrei expansion of [30] and [7] was the expansion of choice. However, in the case of a weakly left $E$-ample monoid, we cannot use this expansion, since it does not respect the ${ }^{+}$operation. The original Szendrei expansion of $[7,30]$ has already been generalized to the weakly left ample case by Gomes [10]. We make the further generalization to the weakly left $E$-ample case. Note that these generalized Szendrei expansions can be applied to semigroups; the original Szendrei expansion could only be applied to monoids.

Definition 4.2. Let $S$ be a weakly left $E$-ample semigroup. We define the Szendrei expansion of $S$ to be the set

$$
\mathrm{Sz}(S)=\left\{(A, a) \in \mathcal{P}^{f}(S) \times S: a, a^{+} \in A \text { and } A \subseteq\left(\widetilde{R}_{E}\right)_{a}\right\},
$$

together with the operation

$$
(A, a)(B, b)=\left((a b)^{+} A \cup a B, a b\right),
$$

where $\mathcal{P}^{f}(S)$ denotes the collection of all finite subsets of $S$ and $\left(\widetilde{R}_{E}\right)_{a}$ is the $\widetilde{\mathcal{R}}_{E}$-class of $a$.

Note that $\mathrm{Sz}(S)$ has the following idempotents:

$$
E(\mathrm{Sz}(S))=\{(F, f) \in \mathrm{Sz}(S): f \in E(S) \text { and } f F \subseteq F\} .
$$

PROPOSITION 4.3. If $S$ is a weakly left E-ample semigroup, then $\mathrm{Sz}(S)$ is a weakly left $\mathrm{Sz}(E)$-ample semigroup with $(A, a)^{+}=\left(A, a^{+}\right)$, where

$$
\mathrm{Sz}(E)=\{(F, f) \in \operatorname{Sz}(S): f \in E \text { and } f F \subseteq F\} .
$$

PROof. The proof is easily adapted from that of [10, Proposition 3]. 
COROLlary 4.4 [10, Proposition 3]. If $S$ is a weakly left ample semigroup, then $\mathrm{Sz}(S)$ is a weakly left ample semigroup with $(A, a)^{+}=\left(A, a^{+}\right)$.

Observe that if $S$ is an arbitrary monoid, then the above expansion reduces to that of [7, 30]: we regard $S$ as being weakly left $\{1\}$-ample, so that any $A \in \mathcal{P}^{f}(S)$ is

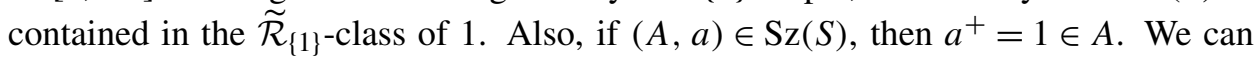
therefore rewrite (4.1) as:

$$
\mathrm{Sz}(S)=\left\{(A, a) \in \mathcal{P}^{f}(S) \times S: 1, a \in A\right\} .
$$

As for multiplication, it is easy to see that we can replace $(a b)^{+}$in (4.2) by 1 , to obtain

$$
(A, a)(B, b)=(A \cup a B, a b),
$$

so $\mathrm{Sz}(S)$ becomes precisely the expansion of [7, 30].

The expansion of Definition 4.2 also generalizes that of [19] for inverse semigroups: if $S$ is inverse, then $\operatorname{Sz}(S)$ is precisely the expansion of [19, Proposition 6.16].

We define a mapping $\eta_{S}: \operatorname{Sz}(S) \rightarrow S$ by $(A, a) \eta_{S}=a$ and note that this is a $(2,1)-$ morphism onto $S$. Moreover, by results of [10], the mapping $\eta_{S}^{*}: S \rightarrow \operatorname{Sz}(S)$, given by $s \eta_{S}^{*}=\left(\left\{s^{+}, s\right\}, s\right)$, is a strong premorphism, so that $\eta_{S}$ is an $F A$-morphism (as defined in the Introduction). For consistency with [13], we will denote $\eta_{S}^{*}$ by $\iota$.

Proposition 4.5. The Szendrei expansion $\mathrm{Sz}(S)$ of a weakly left E-ample semigroup $S$ is generated as a (2,1)-algebra by elements of the form si.

PROOF. We take a general element of $\operatorname{Sz}(S)$ and note that

$$
\begin{aligned}
\left(\left\{s^{+}, s_{1}, \ldots, s_{n}=s\right\}, s\right) & =\left(\left\{s^{+}, s_{1}\right\}, s^{+}\right) \ldots\left(\left\{s^{+}, s_{n}\right\}, s^{+}\right)\left(\left\{s^{+}, s\right\}, s\right) \\
& =\left(\left\{s_{1}^{+}, s_{1}\right\}, s_{1}^{+}\right) \ldots\left(\left\{s_{n}^{+}, s_{n}\right\}, s_{n}^{+}\right)\left(\left\{s^{+}, s\right\}, s\right),
\end{aligned}
$$

since $s_{i} \widetilde{\mathcal{R}}_{E} s$, for each $i=1, \ldots, n$, so

$$
\begin{aligned}
\left(\left\{s^{+}, s_{1}, \ldots, s_{n}=s\right\}, s\right) & =\left(\left\{s_{1}^{+}, s_{1}\right\}, s_{1}\right)^{+} \ldots\left(\left\{s_{n}^{+}, s_{n}\right\}, s_{n}\right)^{+}\left(\left\{s^{+}, s\right\}, s\right) \\
& =\left(s_{1} \iota\right)^{+} \ldots\left(s_{n} \iota\right)^{+}(s \iota),
\end{aligned}
$$

as required.

\section{5. 'Expansion' of partial actions of weakly left $\boldsymbol{E}$-ample semigroups}

At the beginning of the previous section, we stated our desire to find a semigroup $S^{\prime}$ such that if a weakly left $E$-ample semigroup $S$ acts partially on a set $X$, then $S^{\prime}$ is a larger weakly left $E^{\prime}$-ample semigroup for which there is an incomplete action on the same set. It will perhaps come as no surprise that we can take $S^{\prime}$ to be $\operatorname{Sz}(S)$.

We first demonstrate that any strong premorphism between weakly left $E$ - and $F$-ample semigroups may be associated with an $F A$-morphism. 
Proposition 5.1. Let $S$ and $T$ be weakly left $E$ - and $F$-ample semigroups, and let $\theta: S \rightarrow T$ be a strong premorphism. Then there exists a weakly left $G$-ample semigroup $A$, an onto FA-morphism $\phi: A \rightarrow S$ and a (2,1)-morphism $\rho: A \rightarrow T$ such that the following diagram commutes:

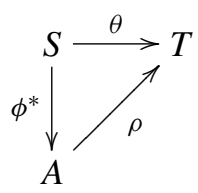

PROOF. It is straightforward to show that $S \times T$ is a weakly left $E \times F$-ample semigroup with $(s, t)^{+}=\left(s^{+}, t^{+}\right)$, for any $(s, t) \in S \times T$. Put $A=\{(s, t): t \leq s \theta\}$. Now if $(s, t),\left(s^{\prime}, t^{\prime}\right) \in A$, then as $\leq$ is compatible with multiplication and $\theta$ is a premorphism,

$$
t t^{\prime} \leq s \theta s^{\prime} \theta \leq\left(s s^{\prime}\right) \theta
$$

so that $(s, t)\left(s^{\prime}, t^{\prime}\right) \in A$. Further, $(s, t)^{+}=\left(s^{+}, t^{+}\right)$; since $t \leq s \theta$,

$$
t^{+} \leq(s \theta)^{+} \leq s^{+} \theta
$$

so that $\left(s^{+}, t^{+}\right) \in A$. Thus $A$ is a $(2,1)$-subalgebra of $S \times T$ and so, by [12, Remark 3.3], $A$ is weakly left $G$-ample, where $G=(E \times F) \cap A$.

Let $\phi: A \rightarrow S$ and $\rho: A \rightarrow T$ be projections; clearly $\phi$ and $\rho$ are $(2,1)$-morphisms with $\phi$ onto. To see that $\phi$ is an $F A$-morphism, observe that $(s, s \theta)$ is the maximum element of $s \phi^{-1}$. Now with $s \phi^{*}=(s, s \theta)$,

$$
\begin{aligned}
\left(s \phi^{*}\right)\left(u \phi^{*}\right) & =(s, s \theta)(u, u \theta) \\
& =(s u, s \theta u \theta) \\
& =\left(s^{+} s u,(s \theta)^{+}(s u) \theta\right) \\
& =(s, s \theta)^{+}(s u,(s u) \theta) \\
& =\left(s \phi^{*}\right)^{+}(s u) \phi^{*},
\end{aligned}
$$

for any $s, u \in S$.

We can now state our 'expansion' result, which we prove using Proposition 5.1; a direct proof appears in the $\mathrm{PhD}$ thesis [14] of the second author.

THEOREM 5.2. Let $S$ and $T$ be semigroups, with $S$ weakly left E-ample and $T$ weakly left $F$-ample, for some $E \subseteq E(S)$ and $F \subseteq E(T)$. If $\theta: S \rightarrow T$ is a strong premorphism, then there exists a unique $(2,1)$-morphism $\bar{\theta}: \operatorname{Sz}(S) \rightarrow T$ such that $\iota \bar{\theta}=\theta$, that is, such that the following diagram commutes:

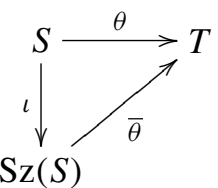


Conversely, if $\bar{\theta}: \mathrm{Sz}(S) \rightarrow T$ is a (2,1)-morphism, then $\theta=\iota \bar{\theta}$ is a strong premorphism.

PROOF. Let $\theta: S \rightarrow T$ be a strong premorphism of the weakly left $E$-and $F$-ample semigroups $S$ and $T$. By Proposition 5.1 we have a weakly left $G$-ample semigroup $A$, an $F A$-morphism $\phi: A \rightarrow S$ and a (2,1)-morphism $\rho: A \rightarrow T$ such that $\theta=\phi^{*} \rho$.

We call upon [10, Theorem 11], adapted to the weakly left $E$-ample case, to guarantee the existence of a $(2,1)$-morphism $\psi: \operatorname{Sz}(S) \rightarrow A$ such that $\psi \phi=\eta_{S}$. Moreover, $\psi$ preserves maxima, in the sense that, for any $s \in S, s \iota \psi=s \phi^{*}$. Consider now the (2,1)-morphism $\psi \rho: \operatorname{Sz}(S) \rightarrow T$; clearly

$$
\iota \psi \rho=\phi^{*} \rho=\theta
$$

so $\psi \rho$ is our required $\bar{\theta}$.

Suppose now that there is another (2,1)-morphism $\sigma: \operatorname{Sz}(S) \rightarrow T$ such that $\iota \sigma=\theta$. Then, for all $s \in S, s \iota \bar{\theta}=s \iota \sigma$, so $\bar{\theta}$ is uniquely defined on elements of the form $s \iota$. By Proposition 4.5 , we deduce that $\bar{\theta}$ is unique.

Conversely, let $\bar{\theta}: \operatorname{Sz}(S) \rightarrow T$ be a $(2,1)$-morphism and let $\theta=\iota \bar{\theta}$. As argued in [10, proof of Theorem 11], $\iota$ is a strong premorphism. Since $\bar{\theta}$ is a $(2,1)$-morphism, it follows easily that $\theta$ is a strong premorphism as required.

If $S$ is an arbitrary monoid (weakly left $\{1\}$-ample), then, by the observations following Definition 3.7 and Corollary 4.4, this theorem reduces to [13, Theorem 4.1].

We will modify the terminology of [13] and refer to this procedure for replacing a partial action by an incomplete action as the expansion of the partial action.

We end this section by specializing to the inverse case, previously considered in [19]. Note that from Lemma 3.12, a map between inverse semigroups is an orderpreserving premorphism if, and only if, it is a strong premorphism. Moreover, any morphism between inverse semigroups preserves ${ }^{+}$.

Corollary 5.3 [19, Proposition 6.20]. Let $S$ and $T$ be inverse semigroups. Then all order-preserving inverse semigroup premorphisms are of the form $\iota \varphi$, where $\varphi: \mathrm{Sz}(S) \rightarrow T$ is a morphism. Consequently, all partial actions of inverse semigroups on a set $X$ are of the form $\iota \varphi$, where $\varphi: \mathrm{Sz}(S) \rightarrow \mathcal{I}_{X}$ is a morphism.

\section{Augmented actions}

We turn now to an analogue of the notion of 'globalization' for the partial actions of weakly left $E$-ample semigroups. The globalization method of [13, 22] does not, in general, respect the ${ }^{+}$operation. If ${ }^{+}$is to be preserved, then we must construct an incomplete action, rather than a global action. We do this via the following definition, a modification of the notion of a 'globalization'.

Definition 6.1. Let $S$ be a weakly left $E$-ample semigroup and let $\theta: S \rightarrow \mathcal{P} \mathcal{T}_{X}$ be a strong premorphism. 
An augmented action for $\theta$ is a pair $(\iota, \varphi)$ consisting of an injection $\iota: X \rightarrow Y$, for some $Y \supseteq X$, and a (2,1)-morphism $\varphi: S \rightarrow \mathcal{P} \mathcal{T}_{Y}$ such that $s \theta=\iota(s \varphi) \iota^{-1}$, for each $s \in S$. The strong partial action of $S$ on $X$, as defined by $\theta$, is said to be a restriction of the incomplete action of $S$ on $Y$, as defined by $\varphi$ (we are here resisting the temptation to call $(\iota, \varphi)$ the 'incompletion' of $\theta$ !).

Note that in moving from [13, Definition 5.1] to Definition 6.1, we have dropped all reference to the generating set, in the interests of simplicity.

For the rest of this section, $S$ will be a weakly left $E$-ample semigroup and $\theta: S \rightarrow \mathcal{P} \mathcal{T}_{X}$ will be a strong premorphism. By Lemma 3.10(2), $E \theta \subseteq E_{X}$.

Our first step in the construction of an augmented action for $\theta$ is to adjoin an identity 1 to $S$ to obtain a weakly left $E^{1}$-ample monoid $S^{1}$. By Lemma 3.11, we have a strong unitary premorphism $\theta^{\prime}: S^{1} \rightarrow \mathcal{P} \mathcal{T}_{X}$, defined by $1 \theta^{\prime}=I_{X}$ and $s \theta^{\prime}=s \theta$, for all $s \in S$.

Switching now to the partial action associated with $\theta^{\prime}$, we define the subset $U$ of $X \times S^{1}$ by

$$
U=\left\{(x, t): \exists x \cdot t^{+}\right\}
$$

Observe that $(x, 1) \in U$ for all $x \in X$.

We define the relation $\sim$ on $U$ by

$$
(x, v u) \sim(x \cdot v, u) \Leftrightarrow \exists x \cdot v \quad \text { and } \quad \forall e \in E^{1}\left[\exists x \cdot(v u e)^{+} \Leftrightarrow \exists(x \cdot v) \cdot(u e)^{+}\right] .
$$

Let $\simeq$ be the equivalence relation generated by $\sim$, and, following Megrelishvili and Schröder [22], let $\rho$ be the equivalence relation on $X \times S^{1}$ given by

$$
(x, m) \rho(y, n) \Leftrightarrow x \cdot m \equiv y \cdot n,
$$

where the symbol $\equiv$ denotes 'strong equality', that is, each side is defined only if the other is, in which case they are equal. Suppose that $(x, v u),(x \cdot v, u) \in U$ with $(x, v u) \sim(x \cdot v, u)$. Since $\exists x \cdot v$, then

$$
\exists x \cdot v u \Longleftrightarrow \exists(x \cdot v) \cdot u,
$$

by (PA2). Hence, $(x, v u) \rho(x \cdot v, u)$, so $\sim \subseteq \rho$. Since $\simeq$ is defined to be the smallest equivalence relation containing $\sim$, then $\simeq \subseteq \rho$.

LEMMA 6.2. If $\exists x \cdot v$, then $(x, v) \sim(x \cdot v, 1)$.

Proof. We know that $(x \cdot v, 1) \in U$. Also, if $\exists x \cdot v$, then $\exists x \cdot v^{+}$, so $(x, v) \in U$.

Suppose that $\exists(x \cdot v) \cdot(1 e)^{+}$, for any $e \in E$. Then $\exists x \cdot v e$, by (PA2), hence $\exists x \cdot(v e)^{+}$.

Conversely, we suppose that $\exists x \cdot(v e)^{+}$. Then $\exists\left[x \cdot(v e)^{+}\right] \cdot v$, since $\exists x \cdot v$ and $x \cdot(v e)^{+}=x$. Hence, $\exists x \cdot(v e)^{+} v$ by (PA2). By the left ample identity, this is equal to $x \cdot v e$. Then, again by (PA2), $\exists(x \cdot v) \cdot e$, or $\exists(x \cdot v) \cdot(1 e)^{+}$, as required. 
We put $Y=U / \simeq$ and denote the $\simeq$-class of $(x, s) \in U$ by $[x, s]$. We define the mapping $\iota: X \rightarrow Y$ by $x \iota=[x, 1]$.

LEMMA 6.3. The mapping $\iota$ is an injection.

PROOF. Suppose that $x \iota=y \iota$. Then

$$
x \iota=y \iota \Rightarrow(x, 1) \simeq(y, 1) \Rightarrow(x, 1) \rho(y, 1) \Rightarrow x \cdot 1 \equiv y \cdot 1 \Rightarrow x=y,
$$

so $\iota$ is one-one.

We now define $\varphi^{\prime}: S^{1} \rightarrow \mathcal{P} \mathcal{T}_{Y}$ to be the mapping which assigns to each $s \in S^{1}$ a partial transformation $s \varphi^{\prime}$ such that

$$
\operatorname{dom} s \varphi^{\prime}=\left\{[x, m]: \exists x \cdot m^{+}, \exists x \cdot(m s)^{+}\right\} \quad \text { and } \quad[x, m] s \varphi^{\prime}=[x, m s] .
$$

LeMma 6.4. Let $(x, v u),(x \cdot v, u),(x, m),(y, n) \in U$ and let $s \in S$. Then:

(i) if $(x, v u) \sim(x \cdot v, u)$, then

$$
\exists x \cdot(v u s)^{+} \Longleftrightarrow \exists(x \cdot v) \cdot(u s)^{+},
$$

and if $\exists x \cdot(v u s)^{+}$, then $(x, v u s) \sim(x \cdot v, u s)$;

(ii) if $(x, m) \simeq(y, n)$ and $\exists x \cdot(m s)^{+}$, then $(x, m s) \simeq(y, n s)$;

(iii) the partial mapping $s \varphi^{\prime}$ is well defined.

PROOF. (i) Let $s$ be any element of $S$. Then

$$
\begin{aligned}
\exists x \cdot(v u s)^{+} & \Longleftrightarrow \exists x \cdot\left(v u s^{+}\right)^{+} \\
& \Longleftrightarrow \exists(x \cdot v) \cdot\left(u s^{+}\right)^{+}, \text {by definition of } \sim, \\
& \Longleftrightarrow \exists(x \cdot v) \cdot(u s)^{+} .
\end{aligned}
$$

If $\exists x \cdot(v u s)^{+}$, then for any $e \in E$,

$$
\exists x \cdot(\text { vuse })^{+} \Leftrightarrow \exists x \cdot\left[v u(s e)^{+}\right]^{+} \Leftrightarrow \exists(x \cdot v) \cdot\left[u(s e)^{+}\right]^{+} \Leftrightarrow(x \cdot v) \cdot(u s e)^{+} .
$$

Therefore $(x, v u s) \sim(x \cdot v, u s)$.

(ii) This follows from (i) by definition of $\simeq$.

(iii) Suppose that $(x, m) \simeq(y, n)$ and that $[x, m]=[y, n] \in \operatorname{dom} s \varphi^{\prime}$. Then $(x, m s) \simeq(y, n s)$ by (ii), that is, $[x, m s]=[y, n s]$, or $[x, m] s \varphi^{\prime}=[y, n] s \varphi^{\prime}$. Therefore $s \varphi^{\prime}$ is well defined.

LEMMA 6.5. The mapping $\varphi^{\prime}$ is a (2,1,0)-morphism.

PROOF. Since

$$
\operatorname{dom} 1 \varphi^{\prime}=\left\{[x, m]: \exists x \cdot m^{+}\right\}=Y \quad \text { and } \quad[x, m]\left(1 \varphi^{\prime}\right)=[x, m],
$$

then $1 \varphi^{\prime}=1$. 
Notice that

$$
\begin{aligned}
\operatorname{dom} s \varphi^{\prime} & =\left\{[x, m]: \exists x \cdot m^{+} \text {and } \exists x \cdot(m s)^{+}\right\} \\
& =\left\{[x, m]: \exists x \cdot m^{+} \text {and } \exists x \cdot\left(m s^{+}\right)^{+}\right\}=\operatorname{dom} s^{+} \varphi^{\prime} .
\end{aligned}
$$

If $[x, m] \in \operatorname{dom} s \varphi^{\prime}=\operatorname{dom} s^{+} \varphi^{\prime}$, then $\exists x \cdot(m s)^{+}$, so $\left(x,(m s)^{+}\right) \sim\left(x \cdot(m s)^{+}, 1\right)$, by Lemma 6.2. Equivalently, $\left(x,(m s)^{+}\right) \sim(x, 1)$, since $x \cdot(m s)^{+}=x$ because $E \theta \subseteq$ $E_{X}$. Then $[x, 1] \in \operatorname{dom} m \varphi^{\prime}$ since $\exists x \cdot m^{+}$, so $\left(x,(m s)^{+} m\right) \sim(x, m)$ by Lemma 6.4. By the left ample identity, $\left(x, m s^{+}\right) \sim(x, m)$, so $\left[x, m s^{+}\right]=[x, m]$, hence $s^{+} \varphi^{\prime}=I_{\text {dom } s \varphi^{\prime}}$.

Now suppose that $[x, m] \in \operatorname{dom}\left(s \varphi^{\prime}\right)\left(t \varphi^{\prime}\right)$. Then $\exists x \cdot(m s)^{+}$and $\exists x \cdot(m s t)^{+}$, so $[x, m] \in \operatorname{dom}(s t) \varphi^{\prime}$.

Conversely, if $[x, m] \in \operatorname{dom}(s t) \varphi^{\prime}$, then $\exists x \cdot(m s t)^{+}$. But $(m s t)^{+} \leq(m s)^{+}$, so $(m s t)^{+} \theta^{\prime} \leq(m s)^{+} \theta^{\prime}$, that is, $\operatorname{dom}(m s t)^{+} \theta^{\prime} \subseteq \operatorname{dom}(m s)^{+} \theta^{\prime}$, so it follows from $\exists x$. $(m s t)^{+}$that $\exists x \cdot(m s)^{+}$. Hence, $[x, m] \in \operatorname{dom} s \varphi^{\prime}$. Then $[x, m s] \in \operatorname{dom} t \varphi^{\prime}$, since $\exists x \cdot(m s t)^{+}$, so $[x, m] \in \operatorname{dom}\left(s \varphi^{\prime}\right)\left(t \varphi^{\prime}\right)$. Therefore, $\varphi^{\prime}$ is a $(2,1,0)$-morphism.

LEMMA 6.6. For each $s \in S^{1}, s \theta^{\prime}=\iota\left(s \varphi^{\prime}\right) \iota^{-1}$.

Proof. Let $x \in \operatorname{dom} s \theta^{\prime}$. Then $\exists x \cdot s$, hence $\exists x \cdot s^{+}$. Now, $x \iota=[x, 1] \in$ $\operatorname{dom} s \varphi^{\prime}$, since $\exists x \cdot s^{+}$, and $x \iota\left(s \varphi^{\prime}\right)=[x, s]=[x \cdot s, 1]$. So $x \in \operatorname{dom} \iota\left(s \varphi^{\prime}\right) \iota^{-1}$ and $x \iota\left(s \varphi^{\prime}\right) \iota^{-1}=x \cdot s=x\left(s \theta^{\prime}\right)$.

Conversely, if $x \in \operatorname{dom} \iota\left(s \varphi^{\prime}\right) \iota^{-1}$, then $[x, 1] \in \operatorname{dom} s \varphi^{\prime}$ and $[x, s] \in \operatorname{dom} \iota^{-1}$, where $\operatorname{dom} \iota^{-1}=X \iota$, so $(x, s) \simeq(y, 1)$, for some $y \in X$. Then, since $\simeq \subseteq \rho$ and $\exists y \cdot 1$, we conclude that $\exists x \cdot s$. Therefore $x \in \operatorname{dom} s \theta^{\prime}$.

We now restrict $\varphi^{\prime}$ to $S$ to obtain a new mapping $\varphi: S \rightarrow \mathcal{P} \mathcal{T}_{Y}$. It is clear that $\varphi$ is a $(2,1)$-morphism with $s \theta=\iota(s \varphi) \iota^{-1}$, for all $s \in S$. We have shown the following theorem.

TheOREM 6.7. Let $S, X, \theta, Y$, ı and $\varphi$ be as previously defined. Then the pair $(\iota, \varphi)$ is an augmented action for $\theta$.

Suppose that we now let $S$ be an arbitrary monoid, regarded as being weakly left $\{1\}$-ample. In this case, $S=S^{1}$, so $\theta=\theta^{\prime}$ and $\varphi=\varphi^{\prime}$. We have $\exists x \cdot t^{+}=x \cdot 1$, for all $(x, t) \in X \times S$, hence $U=X \times S$. Similarly, our $\sim$ becomes the relation

$$
(x, v u) \sim(x \cdot v, u) \Longleftrightarrow \exists x \cdot v,
$$

which is precisely the $\sim\left(=\sim_{S}\right)$ of [13, Section 5]. Observe, finally, that dom $s \varphi=Y$, for all $s \in S$. We see then that in this case the augmented action constructed above becomes precisely the globalization of [13, Section 5] in the case where $N=S$ that is, that of [22, Section 2].

Returning to the general case, we see from Theorem 6.7 that if $\theta: S \rightarrow \mathcal{P} \mathcal{T}_{X}$ is a strong premorphism, then an augmented action exists for $\theta$. We now seek a converse to this. Suppose that a mapping $\theta: S \rightarrow \mathcal{P} \mathcal{T}_{X}$ is the restriction of some incomplete action, in the sense of Definition 6.1. In other words, there exists a set $Y \supseteq X$, an 
injection $\iota: X \rightarrow Y$ and a (2,1)-morphism $\varphi: S \rightarrow \mathcal{P} \mathcal{T}_{Y}$ such that $s \theta=\iota(s \varphi) \iota^{-1}$, for all $s \in S$. Note that

$$
x \in \operatorname{dom} s \theta \Longleftrightarrow x \in X \cap \operatorname{dom} s \varphi \quad \text { and } \quad x(s \varphi) \in X .
$$

PROPOSITION 6.8. The mapping $\theta$ is a strong premorphism.

PRoOf. Let $s \in S$ and suppose that $x \in \operatorname{dom}(s \theta)^{+}=\operatorname{dom} s \theta$, that is, $x \in X \cap \operatorname{dom} s \varphi$ and $x(s \varphi) \in X$. But $\operatorname{dom} s \varphi=\operatorname{dom}(s \varphi)^{+}=\operatorname{dom} s^{+} \varphi$, since $\varphi$ is a $(2,1)$-morphism, so $x \in X \cap \operatorname{dom} s^{+} \varphi$. Note that $s^{+} \varphi=(s \varphi)^{+}=I_{\operatorname{dom} s \varphi}=I_{\operatorname{dom} s^{+} \varphi}$, so $x\left(s^{+} \varphi\right)=x \in$ $X \cap \operatorname{dom} s^{+} \varphi$. We have shown that $\operatorname{dom}(s \theta)^{+} \subseteq \operatorname{dom} s^{+} \theta$. For $x \in \operatorname{dom}(s \theta)^{+}$,

$$
x\left(s^{+} \theta\right)=x \iota\left(s^{+} \varphi\right) \iota^{-1}=x \iota(s \varphi)^{+} \iota^{-1}=x=x(s \theta)^{+},
$$

hence $(s \theta)^{+} \leq s^{+} \theta$.

Let $s, t \in \bar{S}$. Suppose that $x \in \operatorname{dom}(s \theta)(t \theta)$. Then

$$
\begin{gathered}
x \in \operatorname{dom} s \theta, \\
x(s \theta) \in \operatorname{dom}(t \theta) .
\end{gathered}
$$

From (6.2), we deduce that

$$
x \in X \cap \operatorname{dom} s \varphi \quad \text { and } \quad x(s \varphi) \in X,
$$

by (6.1). From (6.3), we further deduce that

$$
x(s \theta) \in X \cap \operatorname{dom}(t \varphi) \quad \text { and } \quad x(s \theta)(t \varphi) \in X .
$$

Now, since $x(s \varphi) \in X$, then $x(s \theta)=x(s \varphi)$, so $x(s \theta)(t \varphi)=x(s \varphi)(t \varphi) \in X$. But $\varphi$ is a (2,1)-morphism, so $x(s t) \varphi \in X$.

Since $x \in X \cap \operatorname{dom}(s t) \varphi$ and $x(s t) \varphi \in X$, then $x(s \theta)^{+}=x \in \operatorname{dom}(s t) \theta$, so that $x \in \operatorname{dom}(s \theta)^{+}(s t) \theta$. Therefore, $\operatorname{dom}(s \theta)(t \theta) \subseteq \operatorname{dom}(s \theta)^{+}(s t) \theta$. so

Conversely, let $y \in \operatorname{dom}(s \theta)^{+}(s t) \theta$. Then $y \in \operatorname{dom} s \theta$ and $y(s \theta)^{+}=y \in \operatorname{dom}(s t) \theta$,

$$
y \in X \cap \operatorname{dom}(s t) \varphi=X \cap \operatorname{dom}(s \varphi)(t \varphi) \quad \text { and } \quad y(s t) \varphi \in X .
$$

Thus $y(s \varphi)(t \varphi)$ is defined, and, since $y \in X, y(s \theta)(t \varphi)=y(s \varphi)(t \varphi)=y(s t) \varphi \in X$. This, together with the fact that $y(s \theta) \in \operatorname{dom} t \varphi$, gives us $y(s \theta) \in \operatorname{dom} t \theta$, hence $y \in \operatorname{dom}(s \theta)(t \theta)$. Therefore, $\operatorname{dom}(s \theta)^{+}(s t) \theta \subseteq \operatorname{dom}(s \theta)(t \theta)$, so $\operatorname{dom}(s \theta)^{+}(s t) \theta=$ $\operatorname{dom}(s \theta)(t \theta)$.

For $y$ in this domain,

$$
y(s \theta)(t \theta)=y(s \varphi)(t \varphi)=y(s t) \varphi=y(s t) \theta=y(s \theta)^{+}(s t) \theta .
$$

Hence, $(s \theta)(t \theta)=(s \theta)^{+}(s t) \theta$.

We have arrived at the following analogue of [13, Theorem 5.7]. 
THEOREM 6.9. An augmented action exists for a premorphism $\theta: S \rightarrow \mathcal{P} \mathcal{T}_{X}$ if, and only if, $\theta$ is strong.

Thus, all strong partial actions of weakly left $E$-ample monoids arise as restrictions of incomplete actions.

EXAMPlE 4. Consider the (2,1)-morphism of Theorem 2.2. This gives us an incomplete action of a weakly left $E$-ample semigroup $S$ on itself. We 'restrict' this incomplete action to a subset $T \subseteq S$, in accordance with the results of this section: let $\theta: S \rightarrow \mathcal{P} \mathcal{T}_{T}$ be the mapping defined by

$$
\begin{aligned}
x \in \operatorname{dom} s \theta & \Longleftrightarrow x \in T \cap \operatorname{dom} s \phi \quad \text { and } \quad x(s \phi) \in T \\
& \Longleftrightarrow x \in T \cap S s^{+}=T s^{+} \quad \text { and } \quad x s \in T,
\end{aligned}
$$

with $x(s \theta)=x \iota(s \phi) \iota^{-1}$, for all $x \in \operatorname{dom} s \theta$, where $\iota: T \rightarrow S$ is inclusion. By Proposition $6.8, \theta$ is a strong premorphism. In fact, $\theta$ is equivalent to the strong partial action of Example 2.

Finally, we specialize Theorem 6.7 to the inverse case. The reader should compare our result with [9, Theorem 4.9] and the relevant remark in [19, Section 6.5].

THEOREM 6.10. Let $S$ be an inverse semigroup and let $S$ act partially on a set $X$ via an order-preserving inverse semigroup premorphism $\theta: S \rightarrow \mathcal{I}_{X}$. Then there exists a set $Y \supseteq X$ and a morphism $\varphi: S \rightarrow \mathcal{I}_{Y}$ such that $s \theta=\iota(s \varphi) \iota^{-1}$, for all $s \in S$.

Proof. We know from Lemma 3.12 that $\theta$ is a strong premorphism; from Theorem 6.7, $Y$ and $\varphi: S \rightarrow \mathcal{P} \mathcal{T}_{Y}$ exist as required. Lemma 3.13 tells us that $\operatorname{im} \varphi \subseteq \mathcal{I}_{Y}$.

\section{Acknowledgement}

We would like to thank Marcel Jackson for supplying us with the Schein and Trokhimenko references.

\section{References}

[1] A. Batbedat, ' $\gamma$-demi-groupes, demi-modules, produit demi-direct', in: Semigroups Proceedings: Oberwolfach 1979, Lecture Notes in Mathematics, 855 (Springer-Verlag, Berlin, 1981), pp. 1-18.

[2] A. Batbedat and J. B. Fountain, 'Connections between left adequate semigroups and $\gamma$ semigroups', Semigroup Forum 22 (1981), 59-65.

[3] J.-C. Birget and J. Rhodes, 'Almost finite expansions of arbitrary semigroups', J. Pure Appl. Algebra 32 (1984), 239-287.

[4] R. Exel, 'Partial actions of groups and actions of inverse semigroups', Proc. Amer. Math. Soc. 126 (1998), 3481-3494.

[5] J. Fountain, 'A class of right PP monoids', Quart. J. Math. Oxford (2) 28 (1977), 285-300.

[6] - 'Adequate semigroups', Proc. Edinb. Math. Soc. (2) 22 (1979), 113-125.

[7] J. Fountain and G. M. S. Gomes, 'The Szendrei expansion of a semigroup', Mathematika 37 (1990), 251-260. 
[8] J. Fountain, G. M. S. Gomes and V. A. R. Gould, 'A Munn type representation for a class of E-semiadequate semigroups', J. Algebra 218 (1999), 693-714.

[9] N. D. Gilbert, 'Actions and expansions of ordered groupoids', J. Pure Appl. Algebra 198 (2005), 175-195.

[10] G. M. S. Gomes, 'The generalised prefix expansion of a weakly left ample semigroup', Semigroup Forum 72 (2006), 387-403.

[11] G. M. S. Gomes and V. Gould, 'Finite proper covers in a class of finite semigroups with commuting idempotents', Semigroup Forum 66 (2003), 433-454.

[12] V. Gould, '(Weakly) left $E$-ample semigroups', http://www-users.york.ac.uk/ varg1/finitela.ps.

[13] C. Hollings, 'Partial actions of monoids', Semigroup Forum 75 (2007), 293-316.

[14] _ 'Partial actions of semigroups and monoids', PhD Thesis, University of York, 2007.

[15] M. Jackson and T. Stokes, 'An invitation to C-semigroups', Semigroup Forum 62 (2001), 279-310.

[16] _ 'Algebras of partial maps', Proceedings of the Special Interest Meeting on Semigroups and Related Mathematics, University of Sydney, 2005, to appear.

[17] J. Kellendonk and M. V. Lawson, 'Partial actions of groups', Internat. J. Algebra Comput. 14 (2004), 87-114.

[18] M. V. Lawson, 'Semigroups and ordered categories I: the reduced case', J. Algebra 141 (1991), $422-462$.

[19] M. V. Lawson, S. W. Margolis and B. Steinberg, 'Expansions of inverse semigroups', J. Aust. Math. Soc. 80 (2006), 205-228.

[20] E. Manes, 'Guarded and banded semigroups', Semigroup Forum 72 (2006), 94-120.

[21] D. B. McAlister and N. R. Reilly, 'E-unitary covers for inverse semigroups', Pacific J. Math. 68 (1977), 161-174.

[22] M. Megrelishvili and L. Schröder, 'Globalisation of confluent partial actions on topological and metric spaces', Topology Appl. 145 (2004), 119-145.

[23] J. Renshaw, 'Inverse semigroups acting on graphs', in: Semigroups and Languages (World Scientific, River Edge, NJ, 2004), pp. 212-239.

[24] B. M. Schein, 'Restrictively multiplicative algebras of transformations', Izv. Vyš̌. Učebn. Zaved. Mat. 4(95) (1970), 91-102 (in Russian).

[25] _ ' Relation algebras and function semigroups', Semigroup Forum 1 (1970), 1-62.

[26] B. Schweizer and A. Sklar, 'The algebra of functions', Math. Ann. 139 (1960), 366-382.

[27] - 'The algebra of functions II', Math. Ann. 143 (1961), 440-447.

[28] - 'The algebra of functions III', Math. Ann. 161 (1965), 171-196.

[29] - 'Function systems', Math. Ann. 172 (1967), 1-16.

[30] M. B. Szendrei, 'A note on Birget-Rhodes expansion of groups', J. Pure Appl. Algebra 58 (1989), 93-99.

[31] V. S. Trokhimenko, 'Menger's function systems', Izv. Vyš̌. Učebn. Zaved. Mat. 11(138) (1973), 71-78 (in Russian).

VICTORIA GOULD, Department of Mathematics, University of York, Heslington York YO10 5DD, UK

e-mail: varg1@york.ac.uk

CHRISTOPHER HOLLINGS, Centro de Álgebra da Universidade de Lisboa, Avenida Professor Gama Pinto, 2, 1649-003 Lisboa, Portugal

e-mail: cdh500@cii.fc.ul.pt 